المجلد 7 ع

أكتوبر-ديسمبر 1/•r

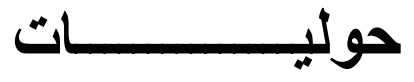

جــــ (ب)

زينب يعوبو مجيد الجاسم

حسن عبد الهادي

الحمل مجمل عبدالفني

علي محمد رشيد

جنان عبد الكاظم لازم

صبيح مزمل جابر

فاطعة حيلر علي

مياسة وليد طه

الريج بهجت احصد

سليم ياوز جمال

الغراح العثيبي

صلاح محسن جانسم

اريتيكنعان حمودي

وضحة عواد الالضلي

فاطمة علي ابو الحليد

شيماء عمر محمد عبد الواحد

شريهة بنت صالح المنديل

عبد الله حميد العثابي

امثثال كاظم النهيب

مزهر محسن الخهاجي

عادل شابث جابر

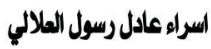

انسام محفند راشّد

لوزي هادي الهنلاوي

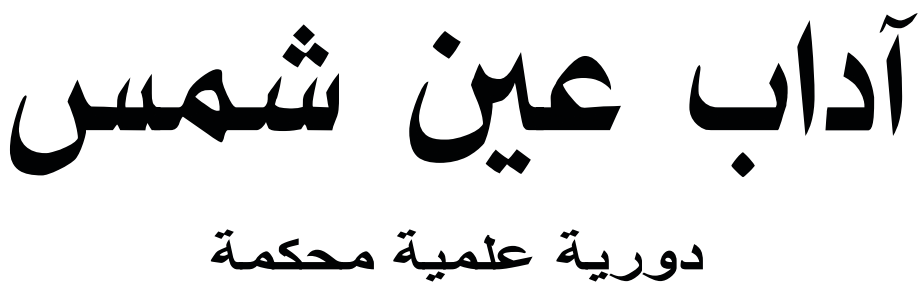

口 بواعد النشّر

البحوث والدراسات المربية

• الصحة الانجابية في الصراق و سبل تنبيتها من خلال المناهيج الدراسية

• المهارات الاتصالية عند معارعي العلادات العامة" واثرها في تمعيل الانشطة الجامعية ( جامعة بغلاد الهوذجا )

•

• عهدة الهبع في نتاجات يوسف حاييم برينر الاببية (نعاذج مغتارة)

• مصفزلت العركة الدكرية في الشرقة الاسلامي وائرها في تعليم المراة

• المزعات الهنية في بلدايات السرد الروائي اليعني

•

• واو الهاصلة جواب التصابل

• الخلمات التعليمية وسيلة للتنمية البشرية

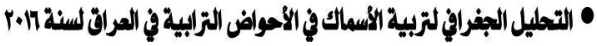

• المشاركة السياسية للمراة الكويتية دراسلة تحليلية

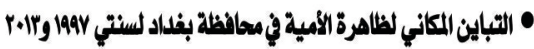

• البنى السردية في شعر الرصائ الاجتماعي

• التوجيه الدلالي لالاليب التصبير في الحرآن الكريم دراسة تطبيهية

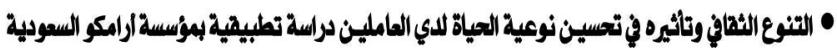

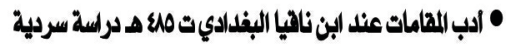

• حجات السلطان الظاهر بييرس والسلطان الناصر محمد بن هلاوون وآثارها على مكة

• التطورات اللداخلية في لططر في عهد الشيخ علي بن عبد الله آل ثاني 1989-1979

• مقاربة تاريخية وسياسية وحضارية في نتائج الاحتلالين الهكبوسي والكشي المصروالعراة

• الاثر البيني لحهر الابار النهطية في تلوث الهواه بالداهيق العالهة (دراسة تطبيهية في محالطظة البصرة)

• تجليات اللفة الثاعرة في الثصن السياسيخ الكاخر عند العمل مطر - هراهة تحليلية

• هضايا البيئة في الصحاثة اليومية العراهية دراسة تحليلية لموضوعات البيئة المنشورة في ه صحف يومية عراهية

$$
\text { أكتوبر- ديسمبر جــزء (ب) }
$$

\title{
المجلد 7
}




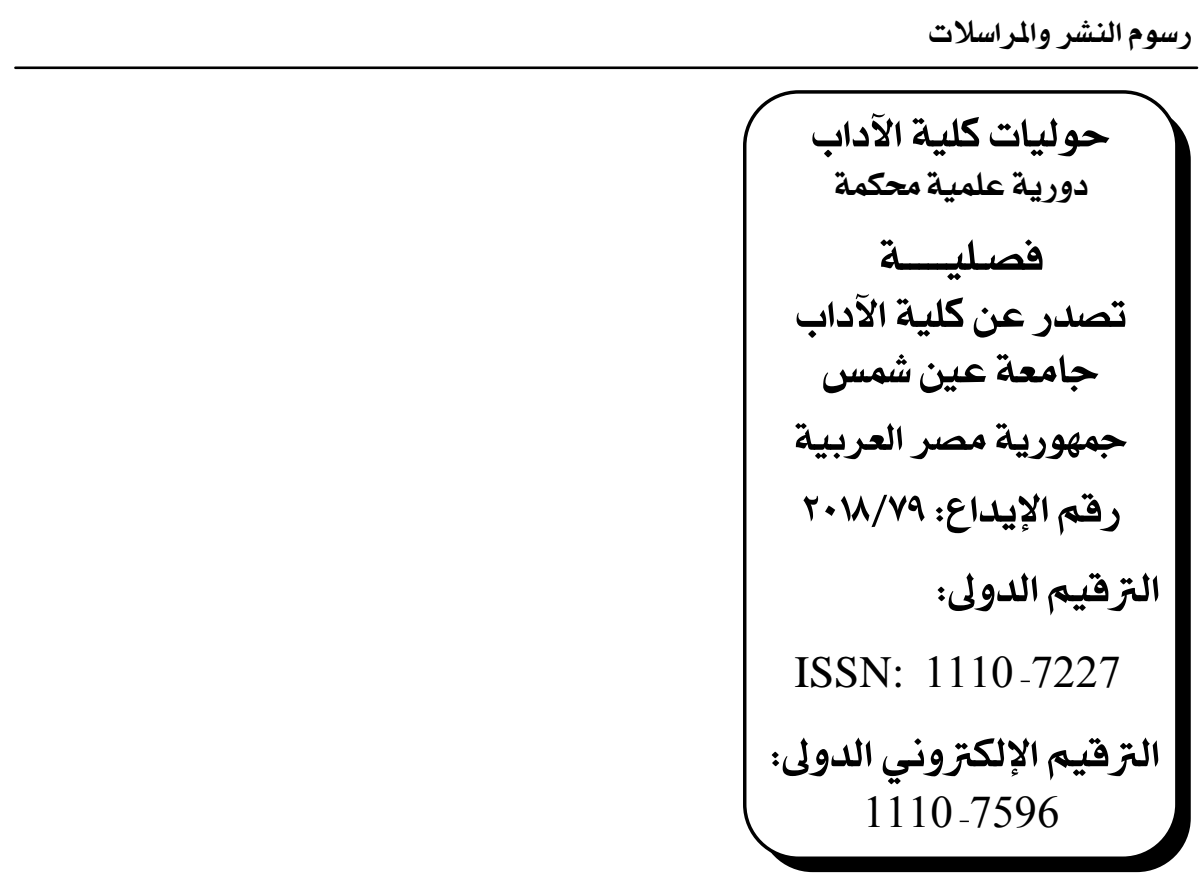

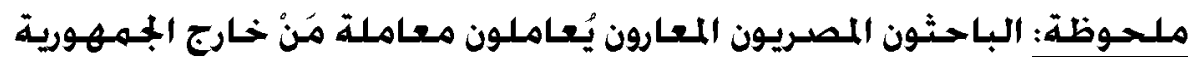

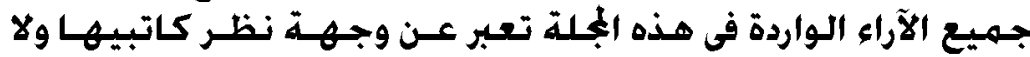

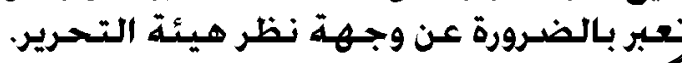

ترسل الطكاتبات باسم أ.د. ردئيس تحرير الحوليات

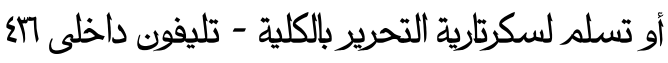

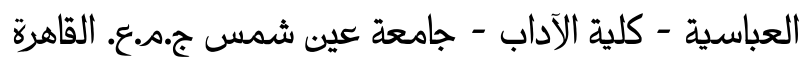

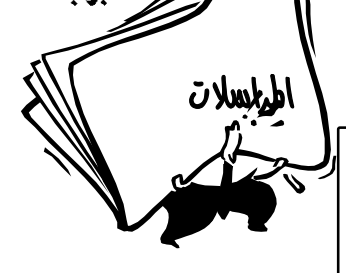

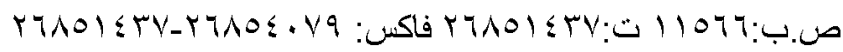

محمول:

Annals_asu@hotmail.com

Annals_asu@yahoo.com 
مجلس الإدارة والمجلس الاستشارى

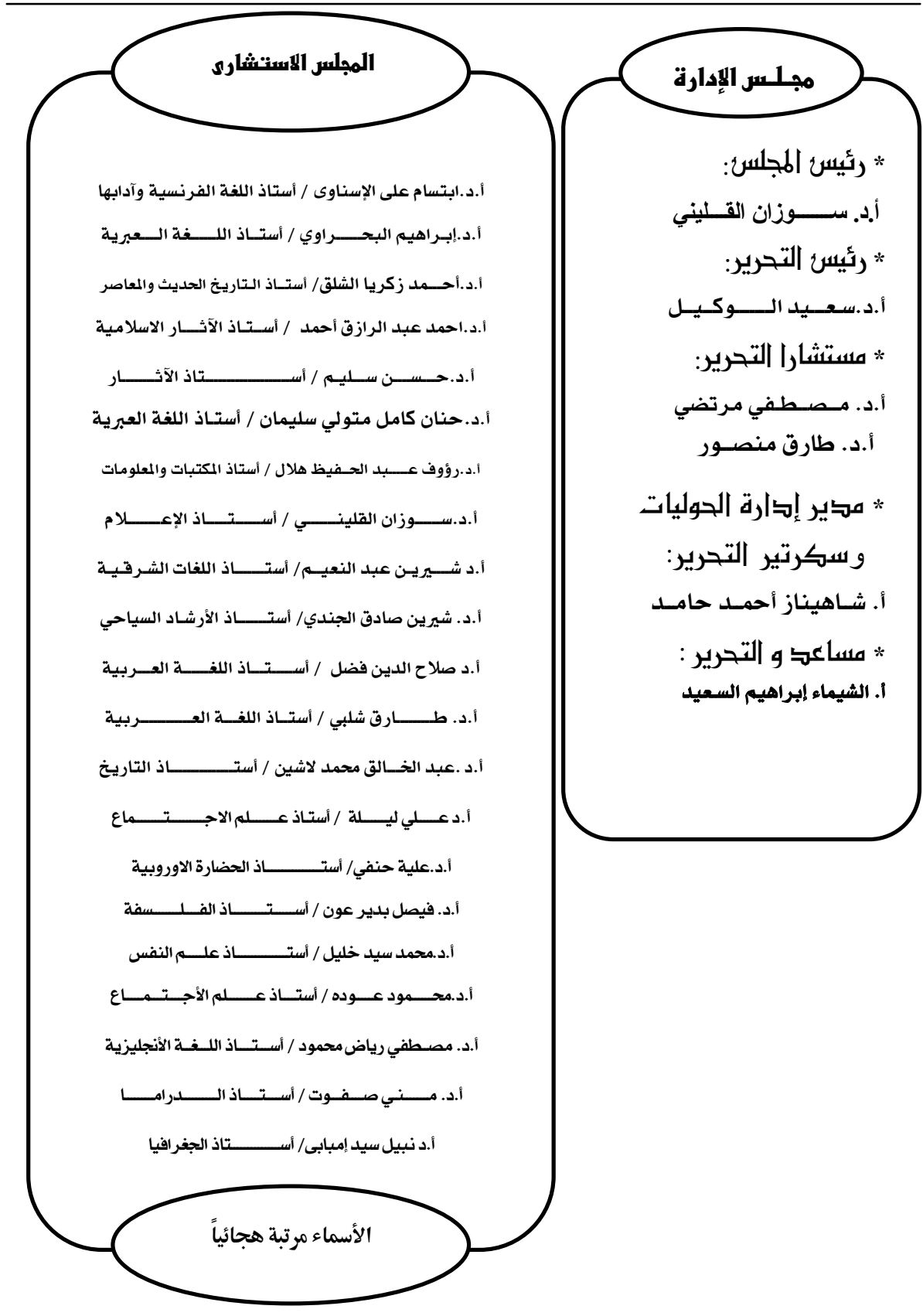

حوليات آداب عين شمس - المجلد 7\$(أكتوبر - ديسمبر 1) +r) 
قيمة الاشتراك

\begin{tabular}{|c|c|c|}
\hline الاشثتراك السنوى & الاشثراك الفصلى & ال الأسعار \\
\hline . جنيه مصري & هب جنيهاً مصرياً للعدد الواحد & أعضاء هيئة التدريس بالنكلية \\
\hline • به جنيه مصري & ج جنيهاً مصرياً للعدد الواحد V. & أفراد (داخل مصر) \\
\hline . عَ جنيهاً مصرياً & •م جنيهاً مصرياً للعدد الواحد & مئسسات (داخل مصر) \\
\hline . بس دو لارأ أمريكياً & .ع دو لاراً أمريكياً للعدد الواحد & مؤسسات (خارج مصر) \\
\hline . . بدو لار أمريكي & هب دو لاراً أمريكياً للعدد الواحد & أفراد (خارج مصر) \\
\hline
\end{tabular}

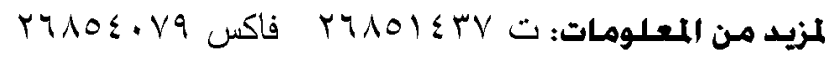

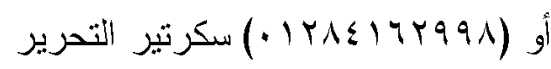

رسبوم النشير : تسدد رسوم التحكيم و المر اجعة عند تسليم البحث.

\begin{tabular}{|c|c|c|c|}
\hline من خارج الجمهورية & من خارج الكلية & أعضاء الكلية & المشترة \\
\hline . ا دولار أمريكي & 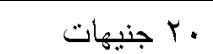 & • أ جنيهات & تكلفة الصفحة الو احدة \\
\hline ، با دولار أمريكي & 0. 0 جنيهاً مصرياً & مك جنيهاً مصرياً & وسوم التمكيم و المر الجعة \\
\hline
\end{tabular}




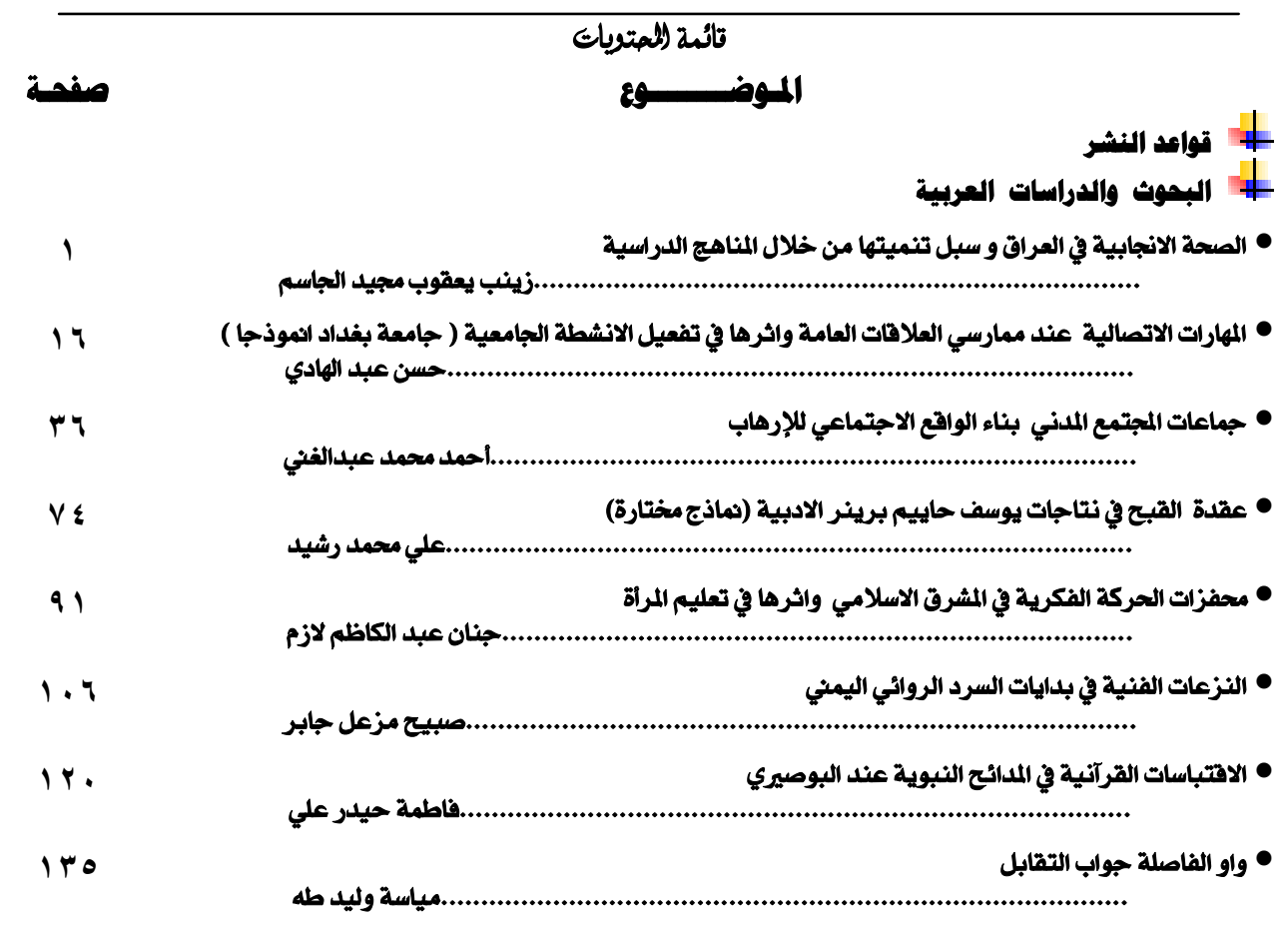

• الخدمات التعليمية وسيلة للتنمية البشرية

• الشاركة السياسية للمراة الكويتية دراسة تحليلية

• التنوع الثقافي وتأثيره في تحسين نوعية الحياة لدي العاملين دراسة تطبيقية بمؤسسة ارامكو السعودية . فاطمة علي ابو الحديد. .

• ابب المقامات عند ابن ناهيا البغدادي ت هAع هـ دراسة سردية شيماء عمر محمد عبد الواحد.

• حجات السلطان الظاهر بيرس والسلطان الناصر محمد بن هلاوون وآثارها على مكة شريفة بنت صالح المنديل

• التطورات الداخلية في فطر في عهد الشيخ علي بن عبد الله آل ثاني 1929-1979 عبد الله حميد العتابي أنسام محمثل راشدل

• مقاربة تاريخية وسياسية وحضارية في نتائج الاحتلاليـن الهكسوسي والكشي لمصر والعراق

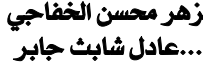

• الاثر البيئي لحفر الابار النفطية في تلوث الهواء بالدهائق العالقة (دراسة تطبيقية في محافظة البصرة)

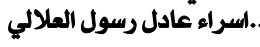
• هضايا البيئة في الصحافة اليومية العراقية دراسة تحليلية لموضوعات البيئة المنشورة في ه صحف يومية عراقية

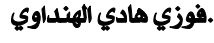





\section{قواعد النشر}

\section{حوليات كلية الآداب - جامعة عين شمس}

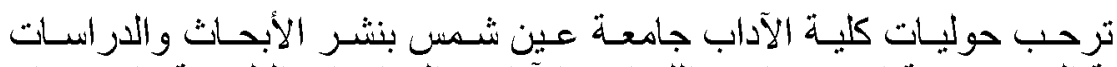

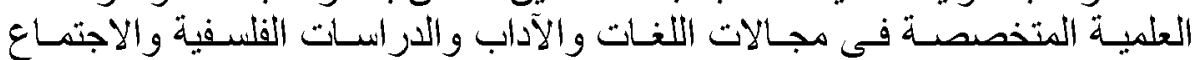

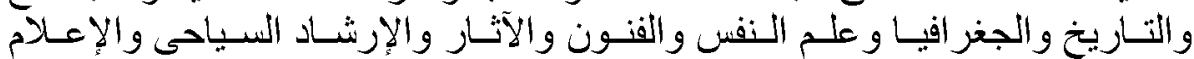
و المكتبات والمعلومات، وذللك وفق للقو اعد التالية:

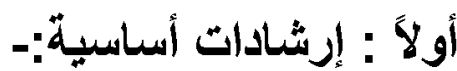

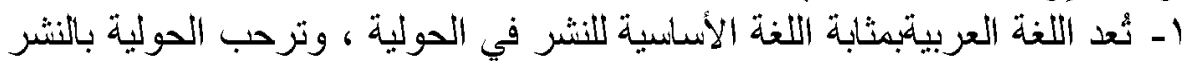

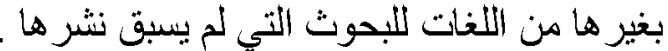

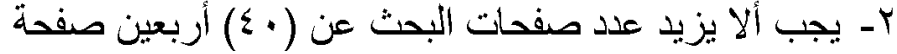

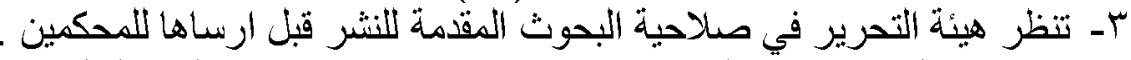

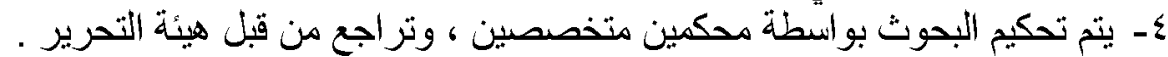

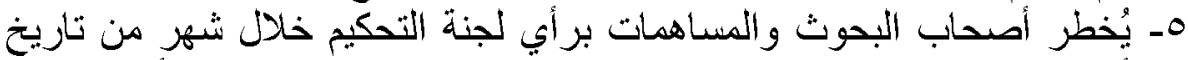
أُستلام البحث ، ويحق للجنة التحكيم طلب أجر اءو تعديلات في الثكل التهل أو المحتوي

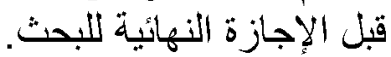

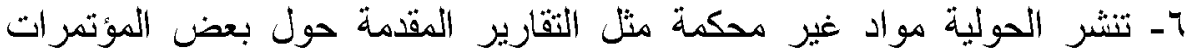
و الندوات والأنثطة الأكاديمية المتعلقة بالمجالات الموضو عية المذكورة أعلاه ،

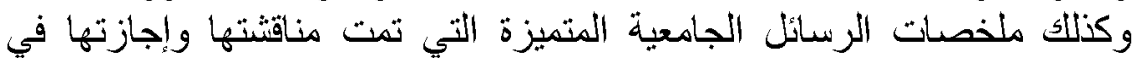

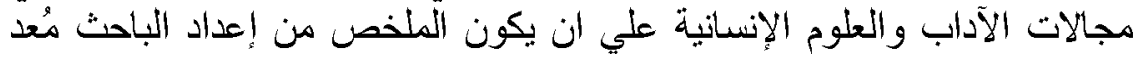

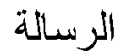

V- الباحث مسئول مسئولية كاملة عن صحة المراجع المستخدمة كما أن هيئة

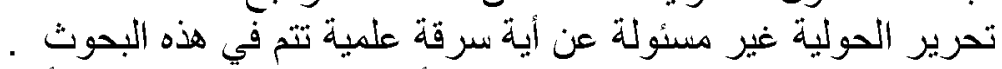

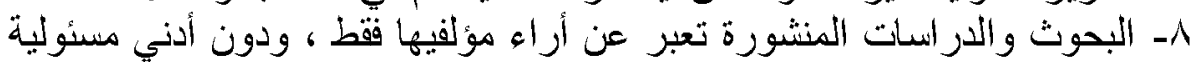

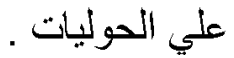

9- المواد المقدمة للنشر يجب ألا يكون قد سبق نشر ها أو تقديمها للنشر إلي جهة أخري . ألخو • ا - لا تنشر المو اد إلا بعد التحكيم وقبولها للنشر وبعد النشر ، لا يسمح بنشر المادة بالثكل نفسة بأي لغة لدي جهات اخري إلا بتصريح كتابي من رئيس هيئة

$$
\text { التحريز. }
$$

ا ـ أصول البحوث التي تقام للحوليات لا ترد سواء نشرت أو لم تنشر . 
r ا ـ يحصل الباحث / الباحثون علي عشر نسخ مستقلة من بحثة (مستلات)مجانا.

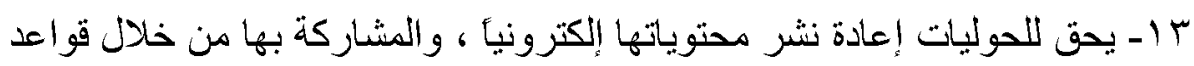

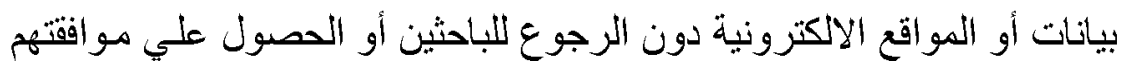
، وذللك دون الاخلال بحقوق الملكية الفكرية للمؤلفين .

ع ا - يوضع تعريف بالباحث في بداية البحث علي أن يثتمل علي : أسم الباحث

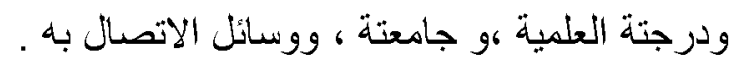

\section{ثانبا: قواعد الكتابة :-}

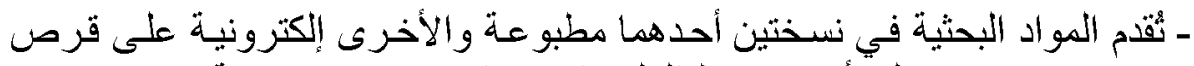

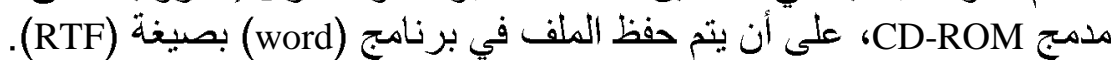

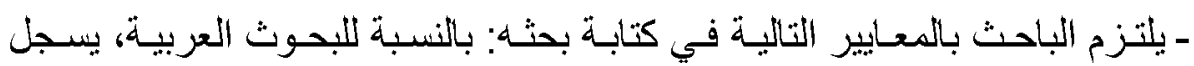

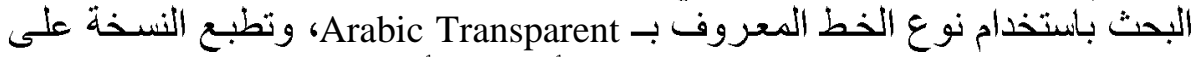

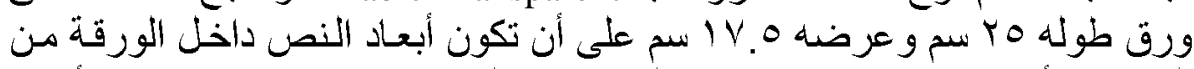

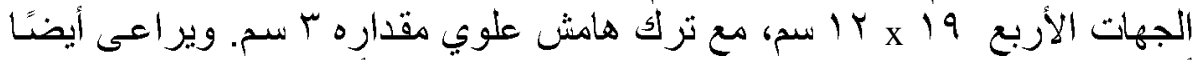

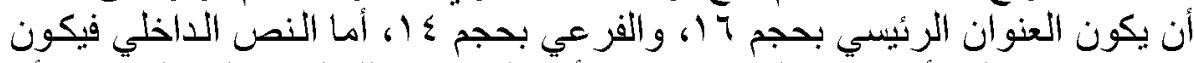

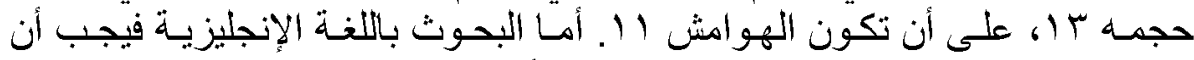

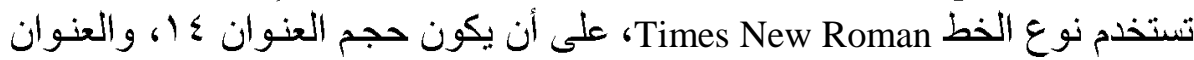

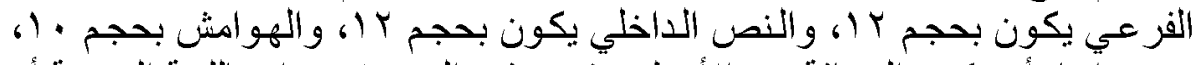

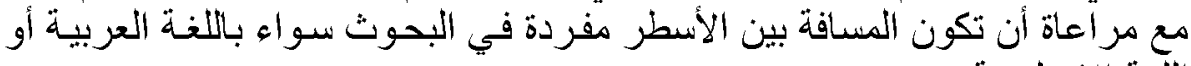
اللغة الإنجليزية. - ثُرسم الخر ائط والأشكال و الرسوم بالحبر الصيني على ورق شفاف (كلك) لتكون

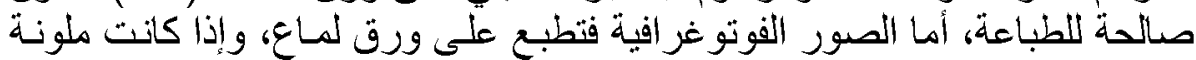
فلابد أن تقدم على الثريحة الثالمة الأصلية.

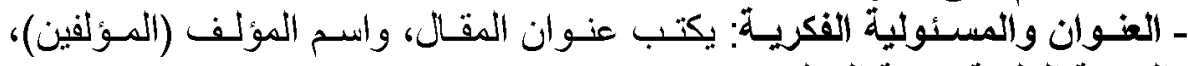

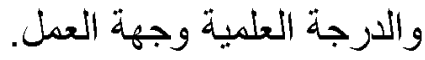

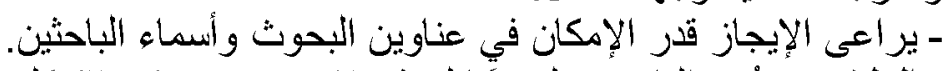

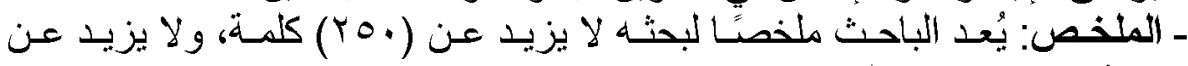

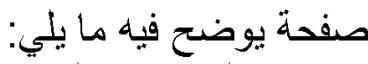
- الموضوع فيه الذي تيناوله الدر اسة وأهدافه.

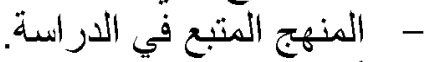

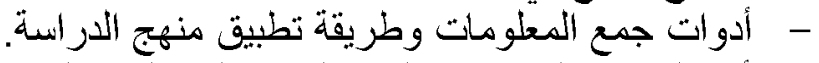

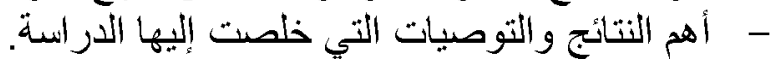




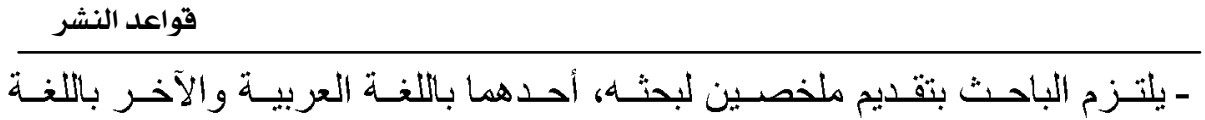

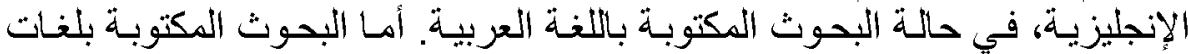

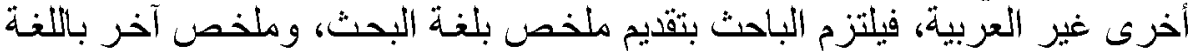

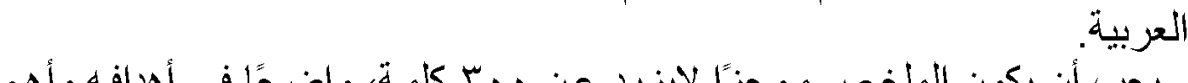

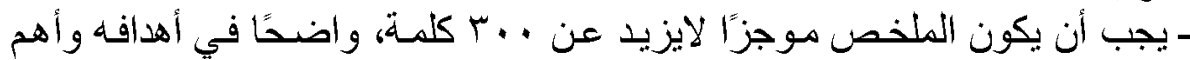

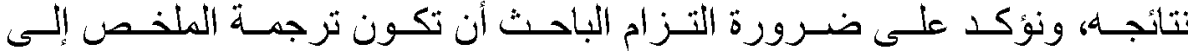

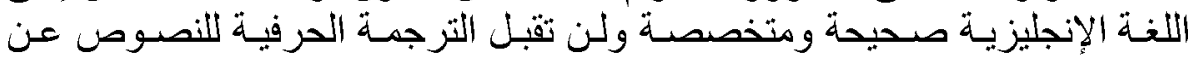
طريق مواقع الترجمة الإليزة ملية على الإنترنت

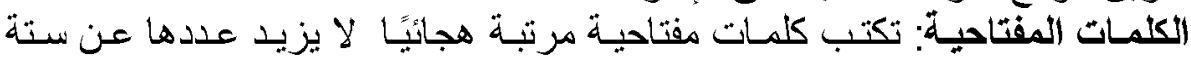
كلمات

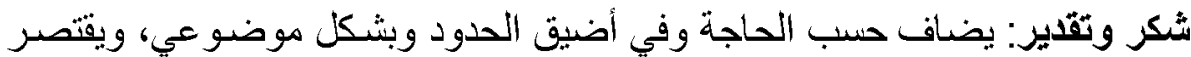

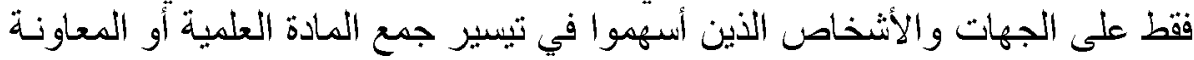

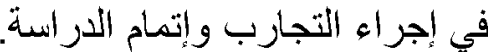

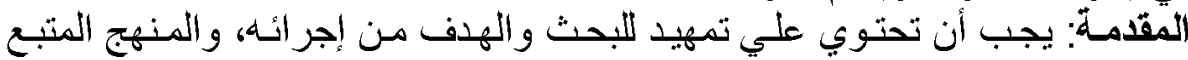

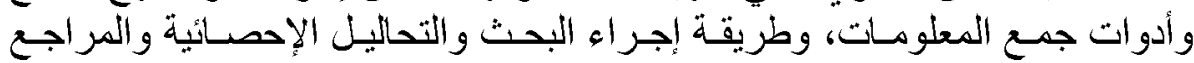

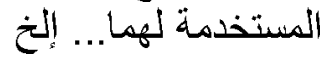

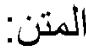

ـ تستخدم فيه ثلاثة مستويات من العناوين أو أقل مع تجنب التذييلات قدر الإمكان.

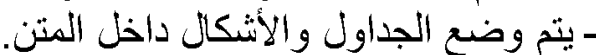

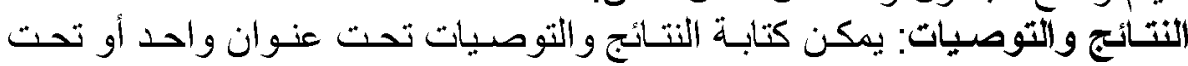

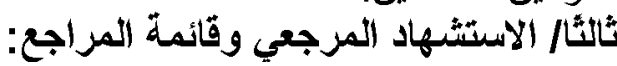

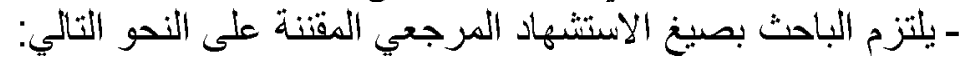

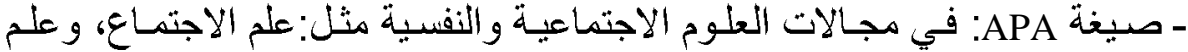

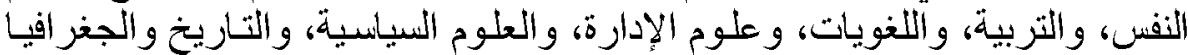
يمكن توثيق الاقتباس في المتن بإحدى طريقتين:

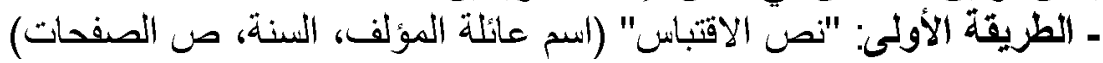

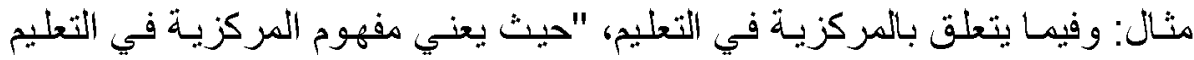

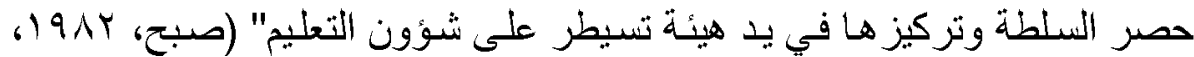


قواعد النشر

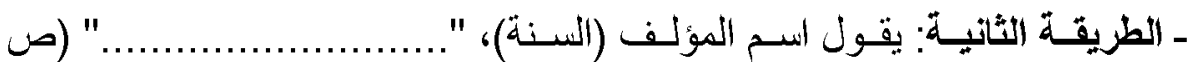

الصفحات)

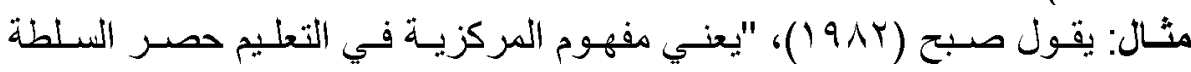

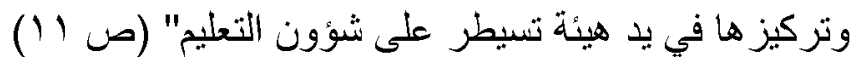

ب- قائمة المصادر والمراجع: ـ ـ الكتب:

اسم العائلة، الاسم الشخصي أو الحرف الأول منه. (السنة). عنوان الكتاب. الطبعة.

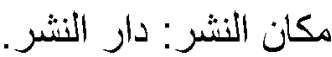
ـ مقالات الدوريات:

اسم العائلة، الاسم الشخصي أو الحرف الأول منه. (السنة). عنوان المقال: العنوان

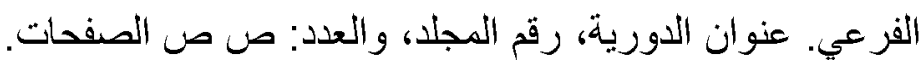
- و صيغة MLA: في الآداب.

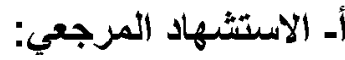
يمكن توثيق الاقتباس في المتن بإحدى طريقتين: ـ الطريقة الأولى: "نص الاقتباس" (اسم عائلة المؤلف ورقم الصفحة)

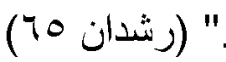
مثال: "...

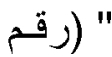

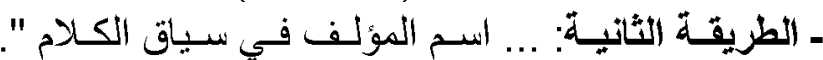
الصفحة مثال: يقول أحمد رشدان "........................ (II (1) بـ قائمة المصادر والمراجع: ـ الكتب:

اسم العائلة، الاسـم الثخصـي أو الحرف الأول منـه. عنوان الكتاب. الطبعة. مكان

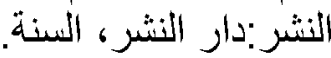

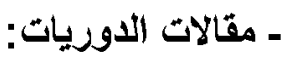

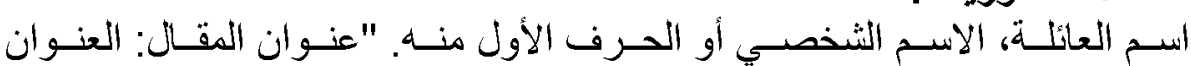
الفر عي." عنوان الدورية رقم المجلد، والعدد (السنة): ص ص الصفي الصفحات. 


\section{ـ ترتيب قائمة المصادر والمراجع:}

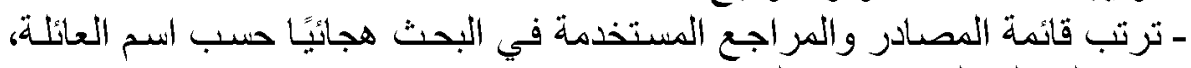

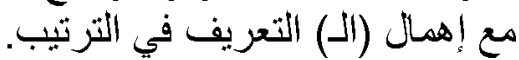

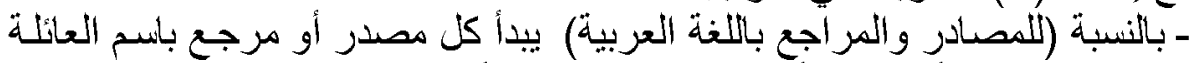

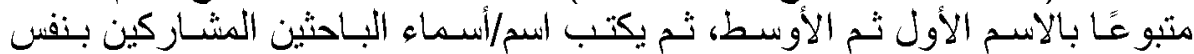
الطريقة

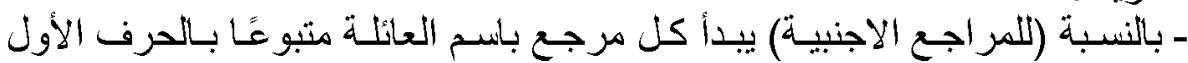

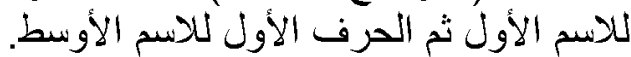

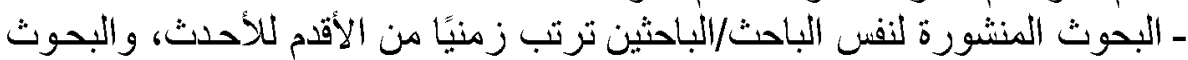
المنشورة في نفس السنة ترتب هجائيًا بالعنوان. 



\section{Annals of the Faculty of Arts \\ Ain shams university}

Volume 46

october - December 2018

Part (B)

\section{A Refereed Scientific periodical}

\section{$\square$ Arabic Researches and Studies:}

- Reproductive Health in Iraq and its Development throughout School Curriculums

- Communication skills of public relations practitioners and their effect on activating university activities (University of Baghdad as a model)

- Civil society groups The construction of the social reality of Terrorism

- The ugliness complex in Yosef Haim Brenner literary Selected exemplars

- Motivations of the intellectual movement in the Islamic Levant And its impact on women's education

- The artistic tendencies in the beginnings of the Yemeni narrative

- Quotations Quraanic in the Prophetic Prophets at Al-Busairi ...

- F. Interval response mapping

- Educational Services and a Means for Human Development

- Geographical Analysis of Fish breeding in Ponds culture in Iraq 2016

- Political participation of Kuwaiti women An analytical study

- The Spatial Variation in Illiteracy in Baghdad for the years 1997,and 2013

- The Narrative Structures inAl Rosafie'sSocial Poetry

- Semantic guidance of the methods of expression in the Koran An Empirical Study

- The Cultural Diversity and its Effect on Improving Labors' Style of Life An Applied Study within Saudi Aramco

- Maqamat literature at Ibn Naqiya al-Baghdadi, 485 AH Narrative study

- pilgrimage Sultan Baybars and Sultan al-Nasir Muhammad And its implications for Mecca

- Internal developments in Qatar during the reign of Sheikh Ali bin Abdullah Al Thani 1949-1960

- A Historical, Political and Cultural Approach In the Results of the Two Occupations Hyksos and Kassite of Egypt and Iraq

- The environmental impact of drilling oil wells in air pollution With Suspended Particles (Applied study in the province of Basra)

- The manifestations of the poet language in the cynical political text When Ahmad Matar - An Analytical Reading

- Environmental issues in the Iraqi daily press Analytical study of environmental issues published in 5 Iraqi daily newspapers
Zaineb Yakoub

Hassan Abd El-Hadi

Ahmed Mohamed Abd- ElGani

Ali Mohamed Rashed

Jinan Abdul Kadhim

Sobeih mazal Jaber

Fatma Haider Ali

Mayassa W. Taha

Areej Bahjat Ahmed

Saleem Y. Jamal

Afrah Al-Otaibi

Salah Mohsen Gasem

AreejKanaan

Wadha Awad Al - Fadhli

Fatma Ali Abulhadid

Shimaa Omar Mohamed Abdel Wahed

Sharifa bint Saleh Al Mandeel

Abdullah Hamid Atabi

Emtsal Kazem

Adil Shabith Jabbir

Mizher Muhssin Al-Khafji

ISRAA ADEL RASOOL AL-ALALI

Ansam Mohammed Rashed

Fawzi Hadi Al - Hindawi

\section{volume 46}

